\title{
EVALUATION OF THE HIGHER TWIST CONTRIBUTION TO THE MOMENTS OF PROTON STRUCTURE FUNCTIONS $F_{2}$ AND $g_{1}$
}

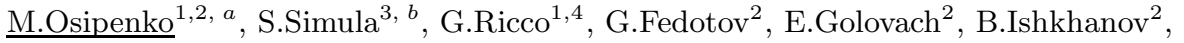 \\ E.Isupov ${ }^{2}$, V.Mokeev ${ }^{5,2}$ \\ ${ }^{1}$ Istituto Nazionale di Fisica Nucleare, Sezione di Genova, 16146 Genova, Italy \\ ${ }^{2}$ Skobeltsyn Institute of Nuclear Physics, 119992 Moscow, Russia \\ ${ }^{3}$ Istituto Nazionale di Fisica Nucleare, Sezione Roma III, 00146 Roma, Italy \\ ${ }^{4}$ Dipartimento di Fisica dell'Università, 16146 Genova, Italy \\ ${ }^{5}$ Thomas Jefferson National Accelerator Facility, Newport News, Virginia 23606 \\ Abstract. We performed the measurement of the inclusive electron scattering off \\ the proton $[1,2]$ in the resonance region $(W<2.5 \mathrm{GeV})$ at momentum transfer \\ $Q^{2}$ below $4.5(\mathrm{GeV} / \mathrm{c})^{2}$ with the CLAS detector. The large acceptance of CLAS \\ provided an access to a large, continuous two-dimensional kinematic domain in $Q^{2}$ \\ and $x$, allowing therefore an integration of the data at fixed $Q^{2}$ over $x$-interval. \\ The covered $x$-interval at each measured $Q^{2}$ value is sufficient for an evaluation \\ of the higher moments $(n>2)$. From these data we extracted the structure \\ function $F_{2}$ and, by including other world data, we studied the $Q^{2}$ evolution of its \\ moments, $M_{n}\left(Q^{2}\right)$, in order to estimate the higher twist contributions. A similar \\ experiment with polarized proton target is completed at CLAS [3]. These new \\ data allow an accurate determination of higher moments of the proton structure \\ function $g_{1}$. A preliminary phenomenological analysis [4] indicates an excess of the \\ higher twist contribution in the spin-dependent structure function with respect to \\ the spin-independent one.
}

\section{Introduction}

Investigation of the nucleon internal structure with electromagnetic probes provided most striking success of the strong interaction theory, QCD. Measured nucleon structure functions give an access to both parton momentum distributions as well as to the scale dependence of the parton coupling with photon. Here the former quantities, parton momentum distributions within the nucleon, are purely phenomenological observable, not derived from the first principles of QCD. Meanwhile, the scale dependence is completely determined by QCD evolution equations. Therefore, in order to compare directly QCD predictions on the nucleon structure to a measurement one has to study the scale dependence of the structure functions avoiding the problem of describing parton momentum distributions, as it was proposed in Ref. $[5,9]$. This can be performed by measuring moments of the structure functions in experiment and studying their scale evolution. In the case of electron-proton scattering it implies a measurement of $Q^{2}$ evolution of the moments of proton structure functions $F_{2}, g_{1}$, $F_{1}$ and $g_{2}$.

\footnotetext{
${ }^{a}$ e-mail: osipenko@ge.infn.it

${ }^{b}$ e-mail: simula@roma3.infn.it
} 


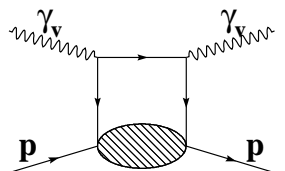

(a)

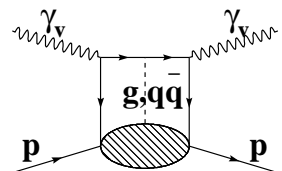

(b)

Figure 1: Twist diagrams:(a) the leading twist contribution evaluated at leading order of pQCD; (b) the contribution of higher twists, where current quark and nucleon remnant can exchange by a system of particles consisting of gluons and $q \bar{q}$-pairs whose complexity is increasing with twist order.

The method of studying moments is based on Operator Product Expansion (OPE) of the virtual photon-nucleon scattering amplitude. This leads to the description of the complete $Q^{2}$ evolution of the moments of the nucleon structure functions. For example, n-th Cornwall-Norton non-singlet moment of the (asymptotic) structure function $F_{2}\left(x, Q^{2}\right)$ for a massless nucleon can be expanded as:

$$
M_{n}^{C N}\left(Q^{2}\right)=\sum_{\tau=2 k}^{\infty} E_{n \tau}\left(\mu, Q^{2}\right) O_{n \tau}(\mu)\left(\frac{\mu^{2}}{Q^{2}}\right)^{\frac{1}{2}(\tau-2)},
$$

where $k=1,2, \ldots, \infty, \mu$ is the factorization scale, $O_{n \tau}(\mu)$ is the reduced matrix element of the local operators with definite spin $n$ and twist $\tau$, related to the non-perturbative nucleon structure. $E_{n \tau}\left(\mu, Q^{2}\right)$ is a dimensionless coefficient function describing the small distance behavior, which can be perturbatively expressed as a power series in the running coupling constant $\alpha_{s}\left(Q^{2}\right)$.

In order to investigate the double expansion in Eq. 1 we truncated both series: in the running coupling constant $\alpha_{s}\left(Q^{2}\right)$ up to Next to Leading Order (NLO) and in twists $\tau$, suppressed by a power of $\left(\mu^{2} / Q^{2}\right)^{\frac{1}{2}(\tau-2)}$, up to $\tau=6$ term. This choice is limiting the analysis to the kinematic region where these parameters are small. We fixed the value of local operator $O_{n \tau}(\mu)$ matrix element at large $Q^{2}$, where all next-to-leading twist expansion terms were neglected. After that we explored low- $Q^{2}$ region to determine the contribution of the higher twist terms.

The higher twists are related to quark-quark and quark-gluon correlations, as illustrated by Fig. 1, and should become important at small $Q^{2}$. In contrast to the asymptotically free quarks, the quarks interacting among themselves during the short time of the photon-proton scattering produce the higher twist terms. The importance of studying the multiparton correlations is due to the fact that they are responsible for the phenomenon of confinement and for the dynamical origin of proton mass. 
The recent experiments undertaken in Thomas Jefferson National Accelerator Facility (USA) provide a large amount of data in the energy range not well explored previously. Measurements of inclusive electron scattering off polarized [3] and unpolarized [1,2] proton targets in Hall B with maximum beam energy of $4.5 \mathrm{GeV}$ allowed a precise extraction of the moments of the proton structure functions $F_{2}$ and $g_{1}$ in the range of momentum transfer, $Q^{2}$, from 0.1 up to $4.5(\mathrm{GeV} / \mathrm{c})^{2}$. These and complementary data from Deep Inelastic Scattering (DIS) were analyzed in terms of perturbative QCD (pQCD) evolution equations and OPE to create a complete picture of the proton structure at different scales.

\section{Experimental Moments of the structure functions}

At small $Q^{2}$ values the moments contain non-negligible mass-dependent terms that produce in Eq. 1 additional $M^{2} / Q^{2}$ power corrections (kinematic twists), that mix with dynamical twists under the renormalization-group equations. To avoid these terms, the moments $M_{n}^{C N}\left(Q^{2}\right)$ have to be replaced by the corresponding Nachtmann moments $M_{n}^{N}\left(Q^{2}\right)$ which by the construction allow to keep the form of the twist expansion in Eq. 1 (see also Ref. [6]). The Nachtmann moments of the structure function $F_{2}$ given by:

$$
M_{n}^{N}\left(Q^{2}\right)=\int_{0}^{1} d x \frac{\xi^{n+1}}{x^{3}} F_{2}\left(x, Q^{2}\right)\left[\frac{3+3(n+1) r+n(n+2) r^{2}}{(n+2)(n+3)}\right],
$$

where $r=\sqrt{1+4 M^{2} x^{2} / Q^{2}}$ and $\xi=2 x /(1+r)$. In the polarized case situation is more involved since Nachtmann moments of the structure function $g_{1}$ depend on both $g_{1}$ and $g_{2}$ :

$$
\begin{aligned}
M_{n}^{N}\left(Q^{2}\right)=\quad & \int_{0}^{1} d x \frac{\xi^{n+1}}{x^{2}}\left\{g_{1}\left(x, Q^{2}\right)\left[\frac{x}{\xi}-\frac{n^{2}}{(n+2)^{2}} \frac{M^{2} x^{2}}{Q^{2}} \frac{\xi}{x}\right]-\right. \\
& \left.g_{2}\left(x, Q^{2}\right) \frac{M^{2} x^{2}}{Q^{2}} \frac{4 n}{n+2}\right\},
\end{aligned}
$$

Because of lack of experimental data on $g_{2}$, moments of the polarized structure function $g_{1}$ contain an intrinsic unavoidable model dependence.

The evaluation of experimental moment $M_{n}$ involves the computation at fixed $Q^{2}$ of an integral over $x$ from the structure function $F_{2}$ weighted with n-th power of $x$. The integral over $x$ was computed numerically. In $x$ intervals where data coverage was not complete we applied essentially model independent interpolation method to avoid as much as possible assumptions on $x$-shape of corresponding momentum distributions. This was accomplished by normalizing the interpolation function directly to experimental data located at the 
edges of interpolating interval, independently for each $Q^{2}$ value. Therefore, the obtained $Q^{2}$ evolution of the moments is free of any model assumptions on the interpolating parton momentum distributions. The low- $x$ extrapolation has been handled by using two parameterizations and estimating the difference as the systematic error. The error come out very small, thanks to the low- $x$ data from HERA. One can note that the low- $x$ extrapolation is only important for the lowest moment, while in higher moments, which are of the main interest of this analysis, the low- $x$ part is strongly suppressed by a power of $x$. The analysis of the proton structure function $g_{1}$ was based not on experimental data, but on a parameterization. A more careful data-based analysis is underway now and results obtained here are therefore preliminary.

The moments were obtained with remarkable statistical and systematic precision of the order of a few percent. In particular, higher moments $(n>2)$ have almost $100 \%$ of significant $x$-interval covered by high precision CLAS data and therefore have tiny error bars. This also allowed to extract the value of QCD running coupling constant $\alpha_{S}\left(M_{Z}^{2}\right)$ with good precision [7].

\section{OPE analysis}

The experimental Nachtmann moments were analyzed in terms of the following twist expansion

$$
M_{n}^{N}\left(Q^{2}\right)=L T_{n}\left(Q^{2}\right)+H T_{n}\left(Q^{2}\right),
$$

where $L T_{n}\left(Q^{2}\right)$ is the leading twist moment and $H T_{n}\left(Q^{2}\right)$ is the higher-twist contribution given by $[8]$

$$
H T_{n}\left(Q^{2}\right)=a_{n}^{(4)}\left[\frac{\alpha_{s}\left(Q^{2}\right)}{\alpha_{s}\left(\mu^{2}\right)}\right]^{\gamma_{n}^{(4)}} \frac{\mu^{2}}{Q^{2}}+a_{n}^{(6)}\left[\frac{\alpha_{s}\left(Q^{2}\right)}{\alpha_{s}\left(\mu^{2}\right)}\right]^{\gamma_{n}^{(6)}} \frac{\mu^{4}}{Q^{4}}
$$

here the logarithmic pQCD evolution of the twist- $\tau$ contribution is accounted for by the term of LO-wise form with an effective anomalous dimension $\gamma_{n}^{(\tau)}$ and the matrix element $a_{n}^{(\tau)}\left(O_{n \tau}(\mu)\right.$ in Eq. 1) fixes normalization of the twist- $\tau$ term at large $Q^{2}$.

The leading twist $L T_{n}\left(Q^{2}\right)$ term was calculated in pQCD to NLO as the sum of a non-singlet and singlet terms. Using the decoupling feature in the pQCD evolution [9] of the singlet quark and gluon densities at large $x$ we considered a pure non-singlet evolution for $n>2$. Therefore, for $n>2$ leading twist contain one unknown parameter, the matrix element $O_{n \tau}(\mu)$. In order to (partly) account for the higher perturbative orders of pQCD we used Soft Gluon Resummation (SGR) technique as in [10]. The resummation of soft gluons does not introduce any further parameter in the description of the leading twist. Leading twist normalization parameter as well as the higher-twist parameters 

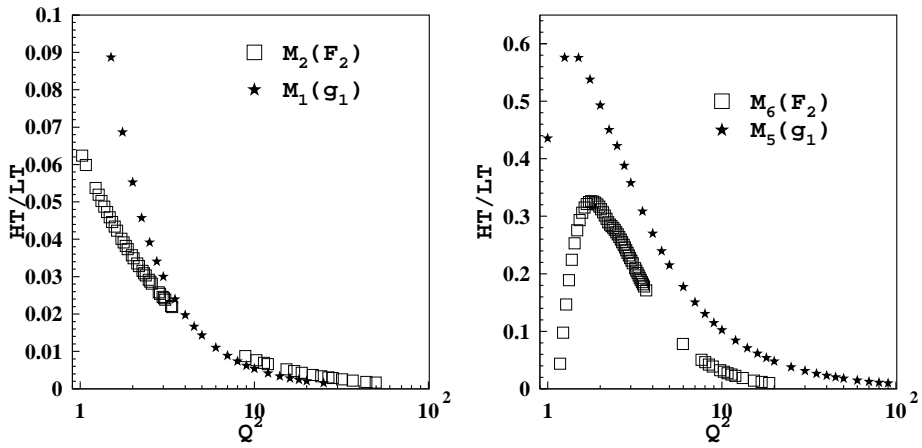

Figure 2: Ratio of the higher to leading twist contributions to the moments $(q(x)$ left panel and $x^{4} q(x)$ right panel) of the proton structure functions $F_{2}$ and $g_{1}$.

$a_{n}^{(4)}, \gamma_{n}^{(4)}, a_{n}^{(6)}, \gamma_{n}^{(6)}$, were simultaneously determined in a $\chi^{2}$-minimization procedure and reported in Refs. [1,4].

The obtained results can be summarized as follows: 1) the contribution of the leading twist remains dominant down to $Q^{2}$ of the order of a few $(\mathrm{GeV} / \mathrm{c})^{2}$; 2) different higher twist terms tend to compensate each other in such a way that their sum is small even in a $Q^{2}$ region where their absolute contributions exceed the leading twist (for details see Ref. [1]); 3) the contribution of higher twists relative to the leading one is very sensitive to the parton polarization. This can be seen in the comparison of the ratio higher to leading twists for structure functions $F_{2}$ and $g_{1}$ shown in Fig. 2. The power of $x$ in the moment is the same for both structure functions, but the ratio is different by almost factor of two at low $Q^{2}$. For the higher $Q^{2}$ values the lowest moment of both structure functions becomes very similar. It gives an idea that the enhancement of the higher twist contribution in $g_{1}$ moments is due to presence of $P_{33}(1232)$ resonance. This excited nucleon state gives strongly negative contribution to the total structure function $g_{1}$ breaking the quark-hadron duality expectations, but it quickly disappears with rising $Q^{2}$ because of the rapid fall-off of the $P_{33}(1232)$ form-factor.

\section{References}

[1] M.Osipenko et al., Phys.Rev. D67, 092001 (2003).

[2] M.Osipenko et al., CLAS-NOTE-2003-001.

[3] R.Fatemi et al., Phys.Rev.Lett. 91, 222002 (2003).

[4] S.Simula, M.Osipenko, G.Ricco and M.Taiuti, Phys. Rev. D65, 034017 (2002).

[5] M.Osipenko et al., in "Frontiers of Particle Physics" (Proceedings of the 10th Lomonosov Conference on Elementary Particle Physics), ed. by A.Studenikin, World Scientific Singapore, 136-141, 2003.

[6] R.Roberts, "The structure of the proton", (Cambridge U.P.), 1993.

[7] S.Simula, M.Osipenko, Nucl.Phys. B675 289 (2003). 
[8] X.Ji and P.Unrau, Phys. Rev. D52, 72 (1995); U.K.Yang and A.Bodek, Phys. Rev. Lett. 82, 2467 (1999).

[9] G.Ricco et al., Nucl. Phys. B 555, 306 (1999).

[10] S.Simula, Phys.Lett. B 493, 325 (2000). 